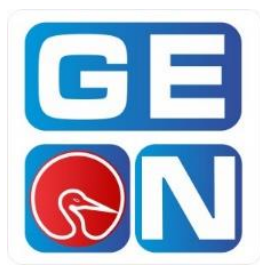

Revista GEON (Gestión, Organizaciones y Negocios.) ISSN: 2346-3910 en línea

revistageon@unillanos.edu.co

Universidad de los Llanos

Colombia

Pérez Paredes, Alfredoi; Cruz de los Ángeles, José Aurelioii;

Gómez Pulido, Andrea Milena.

\title{
Situación actual de la educación financiera en jóvenes universitarios de Villavicencio
}

\section{Colombia}

Revista GEON, Vol. 5, No. 2, 2018

Pág. 115-130

Disponible en: https://doi.org/10.22579/23463910.79

\footnotetext{
${ }^{\mathrm{i}}$ https://orcid.org/0000-0001-8766-5766

ii $\underline{\text { https://orcid.org/0000-0002-8682-366X }}$
}

Esta publicación se encuentra bajo licencia: Creative Commons

ReconocimientoNoComercialSinObraDerivada 4.0 Internacional

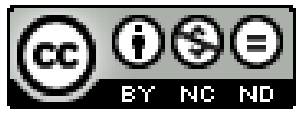

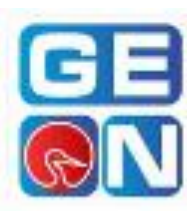

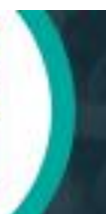

RevistaGEON in $9 f$ 晋 


\title{
Situación actual de la Educación Financiera en Jóvenes Universitarios de Villavicencio Colombia
}

\section{Current situation of Financial Education in Young University Students of Villavicencio Colombia}

\author{
Alfredo Pérez Paredes \\ Alfredoperez2001@hotmail.com
}

Dr. Administración Pública, Benemérita Universidad Autónoma de Puebla BUAP, México

\section{José Aurelio Cruz de los Ángeles \\ Pepejac52@hotmail.com}

Dr. Administración Pública, Benemérita Universidad Autónoma de Puebla BUAP, México

\section{Andrea Milena Gómez Pulido \\ andrea.gomez@unillanos.edu.co}

Administradora de Empresas (e), Universidad de los Llanos, Colombia

Revista GEON Vol. 5 No 2 julio - diciembre 2018

ISSN 2346-3910 en linea http://revistageon.unillanos.edu.co/index.php/geon/article/view/79 


\title{
Resumen
}

La educación financiera, permite a las personas administrar correctamente los recursos y la economía de sus familiares. Al respecto, la OECD sustenta que "la educación financiera es el proceso mediante el cual los individuos adquieren una mejor comprensión de los conceptos y productos financieros y desarrollan las habilidades necesarias para tomar decisiones informadas, evaluar riesgos y oportunidades financieras, y mejorar su bienestar". (OECD, 2008). Por tanto, el presente trabajo de investigación persigue el objetivo de medir la educación financiera de los jóvenes universitarios de Villavicencio Colombia. Se aplicó un instrumento compuesto de 18 ítems, que permite identificar el conocimiento que se posee acerca de la educación financiera. Parte de los resultados conseguidos nos manifiesta que, del total de la población de alumnos encuestados, la mayoría, no tiene claro el tema de la educación financiera.

Palabras Clave: Educación, crédito, ahorros, universitarios, Villavicencio

\begin{abstract}
Financial education allows people to properly manage the resources and finances of their family members. In this regard, the OECD argues that "financial education is the process by which individuals acquire a better understanding of financial concepts and products and develop the necessary skills to make informed decisions, assess risks and financial opportunities, and improve their well-being". (OECD, 2008). Therefore, this research work pursues the objective of measuring the financial education of university students in Villavicencio Colombia. An instrument composed of 18 items was applied, which allows to identify the knowledge that is possessed about financial education. Part of the results obtained shows us that, of the total population of students surveyed, the majority is not clear about the subject of financial education.
\end{abstract}

Keywords: Education, Credit, Savings, University, Villavicencio

\section{Introducción}

Colombia, al igual que otros países de Latinoamérica como México, demanda un Sistema Financiero saludable que apruebe el buen funcionamiento y desarrollo de la economía en la que se hallan, y de esta manera, beneficie el crecimiento económico sostenido y el bienestar de la sociedad, por lo que se requiere de la 
intervención del marco regulatorio de las distintas leyes sobre las que normaliza sus operaciones, así como de la inspección que avale la entereza del sistema y se salvaguarden los intereses del público.

Un instrumento primordial que promueve el desarrollo del sistema es la educación financiera, pues condesciende una toma de decisiones congruente que beneficia a la población, lo cual se manifiesta en la aplicación adecuada de los servicios financieros y servicios de crédito que brinda la banca de México.

En la actualidad, algunos países de Latinoamérica cuentan con un porcentaje mínimo en comparación a otros países en lo que compete a educación financiera. En México, la educación financiera ha sido relegada durante muchos años; mientras en otras naciones, desde hace décadas, se ha integrado este tipo de educación en los programas educativos, en nuestro país apenas se está analizando cómo introducirla a las escuelas. De acuerdo con datos de la Comisión Nacional para la Protección y Defensa de los Usuarios de Servicios Financieros, cerca de 62 de cada 100 mexicanos carece de educación financiera. (CONDUSEF, 2010)

El actual trabajo de investigación tiene por objeto medir el conocimiento que se tiene sobre la educación financiera, así como el impacto que éste crea en sus finanzas individuales al decidir consumir algunos de los servicios financieros, y si es necesario, educar con algunas metodologías que les permitan negociar la decisión sobre los productos y servicios financieros que se ofrecen. Dicho estudio se aplica a los jóvenes universitarios de Villavicencio, departamento Meta, Colombia.

Subsiguientemente se presentan conceptos de los términos vinculados a los servicios financieros y de crédito, la educación financiera, el endeudamiento y la toma de decisiones que se derivan de obtener finanzas particulares sanas.

En continuidad con el progreso de la investigación, se encuentra la metodología manipulada, se exteriorizan los resultados y para finalizar las conclusiones obtenidas, que nos decretarán si es necesario capacitar a los jóvenes en lo referente a la educación financiera que contribuya a su bienestar personal.

El hacer hincapié a la educación financiera en jóvenes de educación superior abrirá paso a la toma de decisiones apropiadas que impacten positivamente finanzas personales, reduciendo la incertidumbre, así como el allegarse de otros medios informales al intentar captar ingresos que cubran sus objetivos de corto, mediano y largo plazo.

\section{Marco Contextual}

El municipio de Villavicencio pertenece a la región de la Orinoquia Colombiana, es la capital del departamento del Meta, conocido por su clima cálido, la tradicional mamona y el joropo.

La Ultima población conocida es 506 000 (Año 2017). Corresponde al 1.031\% de la total población colombiana, según (unidad administrativa: Meta) 
La economía de Villavicencio depende principalmente de la agricultura, la ganadería y la minería, aunque en los últimos años el sector comercio ha fortalecido el desarrollo económico, esto obedece a la dinámica de comunicación de sus vías, Villavicencio es el paso obligado para la mayoría de las cosechas y de ganado que producen los departamentos aledaños, que van hacia el interior y el centro del país.

Villavicencio es el principal centro urbano universitario de la región al cual llegan jóvenes de los departamentos vecinos, Como ciudad eje de los Llanos Orientales, Villavicencio cuenta con una red de instituciones educativas de carácter público y privado, algunas de ellas son:

Universidad de los Llanos: única institución académica de educación superior, de carácter público, es la más grande de la región, ubicada en Villavicencio, su sede principal está ubicada en la vereda Barcelona y cuenta con una segunda sede (san Antonio), ubicada en el centro de la ciudad, algunos de los programas que oferta son: administración de empresas, Ingeniería Agronómica, Medicina Veterinaria y Zootecnia, Enfermería, Licenciatura en Educación Física, Ingeniería Electrónica, Economía entre otras. Además, cuenta con varios programas de especialización y maestrías.

Existen algunas otras como lo son: Universidad Santo Tomás, Escuela Superior de Administración Pública, Universidad Nacional Abierta y A Distancia, Corporación Universitaria del Meta, Corporación Universitaria Minuto de Dios, Fundación Universitaria San Martín, Corporación Universitaria Remington y Corporación Unificada Nacional de Educación Superior - CUN.

Lo anterior refleja la importancia que tiene Villavicencio al contar con varias Instituciones de Educación Superior y es por ello por lo que la población existente de jóvenes universitarios se convierte en el objeto de estudio.

\section{Marco Teórico}

Sistema Financiero Colombiano. José Darío Uribe expresa que "el sistema financiero colombiano está conformado por los establecimientos de crédito (EC), las entidades de servicios financieros (ESF) y otras entidades financieras, las cuales, en su mayoría, se han agrupado mediante la figura de los conglomerados financieros, haciendo presencia tanto en el ámbito interno como externo. Luego de la crisis financiera de finales de la década de los noventa, este sector se ha venido fortaleciendo gracias, a la regulación del gobierno nacional y de la Superintendencia Financiera de Colombia (SFC), lo que se ha reflejado en buenos indicadores de rentabilidad, riesgo $\mathrm{y}$ solvencia." (Uribe, 2013)

$\mathrm{Su}$ función principal es la de captar recursos legales de los particulares y convertirlos en depósitos a la vista como las cuentas de ahorro o a término como los CDT, para colocarlos en el mercado a través de instrumentos como los préstamos, descuentos, anticipos o cualquier operación de crédito. 
Las principales Instituciones Financieras que existen en Colombia son:

A.Establecimientos de crédito o Bancos: se encargan de ofrecer servicios financieros exclusivos que los diferencian de las demás entidades financieras, como cuentas de ahorro y cuentas corriente, créditos y otros productos financieros entre otros.

Corporaciones Financieras: $\mathrm{Te}$ ofrecen principalmente servicios financieros corporativos y empresariales. $\mathrm{Su}$ función principal corresponde a la captación de recursos a través de depósitos o instrumentos de deuda a plazos como los CDT, con el objetivo de utilizar esos recursos para prestarlos a las empresas, para así fomentar y promover el desarrollo de la empresa colombiana. Por ejemplo, cualquier asociación de productores pequeños o medianos puede acceder a los servicios de financiamiento de las corporaciones financieras.

Compañías de Financiamiento Comercial: $\mathrm{Te}$ ofrecen servicios de inversión, créditos y operaciones de leasing, a través de ellas puedes obtener por ejemplo una maquinaria para tu empresa por un determinado tiempo pagando una cuota de arrendamiento con la opción de comprar esta al finalizar el contrato. (Arboleda, 2010)

B. Otro tipo de Entidades Financieras Fiduciarias: Se encargan de administrar tus bienes, inversiones o proyectos de construcción de vivienda. Para mayor información ingresa

a o Compañías de seguros: Existen 2 tipos, las de seguros de vida que se encargan de cubrir lo relacionado con accidentes y pólizas de vida entre otros, y las de seguros en general tales como los seguros sobre bienes inmuebles, desempleo, automóviles. Para más información ingresa a FASECOLDA.

Sociedades comisionistas de bolsa: Te ofrecen administrar tus ahorros o ganancias e invertirlas en el mercado bursátil, generándote nuevas ganancias sobre tu capital.

Los Servicios Financieros. Se entiende por servicio financiero "al conjunto de instrumentos, mercados e instituciones financieras que corrigen los problemas de información y disminuyen los costos de transacción" (Terceño \& Guercio, 2010), destacando al más importante intermediario financiero: el banco, cuya función vital es recibir dinero para depósitos en cuenta, y ofrecer créditos, informando el costo total que implica al momento de adquirirlo. He ahí la importancia de los bancos dentro de una economía, ya que canalizan el dinero que ahorran las personas, para prestarlo a aquellos individuos que lo necesitan para realizar proyectos de inversión, para el consumo personal o para la adquisición de bienes duraderos como casas, automóviles, enseres domésticos, entre otros.

Otro tipo de servicio financiero son las casas de bolsa, las cuales se dedican a dar servicios para poner en contacto la oferta y la demanda de valores emitidos o garantizados por terceros, (acciones de empresas o títulos de deuda). Estas 
operaciones consisten en la colocación de valores mediante ofertas públicas, así como en la compra, la venta y el préstamo de valores en el mercado de valores. Estos intermediarios están regulados por la Ley del Mercado de Valores, entre otras leyes. Otros intermediarios financieros que otorgan créditos para la obtención de casas, automóviles y demás bienes de consumo duradero, son las sociedades financieras de objeto limitado (SOFOLES) y las sociedades financieras de objeto múltiple (SOFOMES) (BANXICO, 2013).

Ahorro. Significa "reservar" una capacidad productiva, no empleada en la producción de bienes de consumo y dejarla disponible para producir nuevos bienes de inversión.

Otra definición nos indica que "es la parte del capital que reservan y guardan las personas fruto de sus rentas. Es, por tanto, el capital que no se consume o se gasta y que se reserva para cubrir una necesidad o contingencia futura e incluso para dar como herencia a tus herederos legales." (Burdillo Vázquez, 2016) Parafraseando, el ahorro es la parte del ingreso que se designa guardar para el futuro.

La mayor proporción de adultos con cuenta en un banco o institución financiera se encuentran en los que habitan en localidades con más habitantes, los que tienen un nivel de estudios mayor y los que reciben más ingreso por su trabajo, actividad o negocio; lo que no es evidente es una mayor proporción de ahorro formal debido al sexo o al rango de edad. México se caracteriza por ser un país joven, urbano, con un nivel promedio de estudios de secundaria y con más mujeres que hombres. De esta forma, con datos de la ENIF, se estima que alrededor de dos de cada tres adultos tienen una cuenta si poseen de forma conjunta las características sociodemográficas: haber cursado al menos un grado de secundaria, recibir ingresos por su trabajo, actividad o negocio, vivir en una localidad de $100 \mathrm{mil}$ y más habitantes, tener entre 18 y 30 años $\mathrm{y}$ ser mujeres. Lo anterior no necesariamente quiere decir que poseer estos atributos aumenten la probabilidad de tener una cuenta en comparación de otros dentro del mismo atributo (por ejemplo, haber cursado una licenciatura en comparación con secundaria), ya que para ello se necesitaría realizar un análisis probabilístico. (ENIF, Comisión Nacional Bancaria y de Valores MX, 2017)

De igual forma, alrededor de tres de cada cuatro adultos ahorraron a través de mecanismos informales si tienen las siguientes características sociodemográficas: haber cursado al menos un grado de secundaria, percibir un ingreso por su trabajo, actividad o negocio, vivir en una localidad de 100 mil y más habitantes, tener entre 18 y 30 años y ser hombres. (ENIF, Comisión Nacional Bancaria y de Valores MX, 2017)

Inversión. La inversión es todo desembolso de recursos financieros para adquirir bienes concretos durables $\mathrm{o}$ instrumentos de producción, denominados bienes de equipo, y que la empresa utilizará durante varios años para cumplir su objeto social. (Peumans, 1967)

Por tanto, en la inversión hay una serie de elementos que la definen: 
- Un sujeto (Inversor)

- Un activo financiero (Dinero)

- La renuncia a una satisfacción inmediata

- Un activo en el que se invierte

- Una promesa de recompensa futura más o menos incierta

La inversión es financiera cuando el dinero se destina a la adquisición de activos cuyo precio depende de las rentas que se supone generarán en el futuro, como las acciones de una empresa, depósitos a plazo y los títulos de deuda.

Toda inversión implica tanto un riesgo como una oportunidad. Es un riego debido a que la devolución del dinero que invertimos no está garantizada, y es una oportunidad debido a que puede ocurrir que se multiplique el dinero invertido.

En toda inversión hay que considerar cuatro variables distintas pero relacionadas:

1. El rendimiento esperado, es decir, la rentabilidad que esperamos obtener de nuestra inversión, y se suele medir como porcentaje de la cantidad invertida. Aquí hay que contemplar que hay una relación directa entre el rendimiento esperado y el riesgo asumido: a mayor rendimiento, mayor riesgo.

2. El riesgo aceptado, es decir, la incertidumbre sobre el rendimiento y sobre la posibilidad de que no se recupere el dinero invertido. Esta es una variable muy subjetiva y que definirá el perfil del inversor, y la aversión al riesgo que muestre. Un inversor conservador tenderá a invertir en productos de bajo riesgo y, por ende, de menor rentabilidad, como son los títulos de renta fija o los depósitos a plazo, aunque el interés sea menor que el que podría obtener en títulos de renta variable $\mathrm{u}$ otras inversiones.

3. Otra variable es el horizonte temporal de la inversión, que puede ser a corto, medio y a largo plazo. Las inversiones a más largo plazo suelen ofrecer tipos de interés mayores que los plazos menores. Un ejemplo claro son las letras del tesoro frente a los bonos $\mathrm{u}$ obligaciones del estado.

4. Finalmente, un último punto que deberíamos contemplar es la liquidez de nuestra inversión; es decir, con qué rapidez podemos recuperar nuestra inversión y a qué coste en caso de necesitar recuperarla. Por ejemplo, no es lo mismo invertir en acciones de uno de los grandes bancos del país, para los que se negocian diariamente en el mercado continuo miles de títulos, que hacerlo en una empresa cuyo volumen es muy bajo y, por tanto, desprendernos de los títulos podría llevar días, e incluso semanas. (Financiera E., 2017)

Seguros. El seguro es un medio para la cobertura de los riesgos al transferirlos a una aseguradora que se va a encargar de garantizar o indemnizar todo o parte del perjuicio producido por la aparición de determinadas situaciones accidentales.

Es una fórmula eficaz de cobertura que implica pagar una cierta cantidad por una prestación o indemnización futura en caso de que se presente una situación adversa, que, en algunos casos, puede ser extrema. (MAPFRE, 2011) 
Existe un órgano que se encarga de supervisar que la relación entre los sectores asegurador y afianzador y el marco normativo sea lo más transparente posible, ésta es: la Comisión Nacional de Seguros y Fianzas es un Órgano Desconcentrado de la Secretaría de Hacienda y Crédito Público, asimismo preserva la solvencia y estabilidad financiera de las instituciones de Seguros y Fianzas, para garantizar los intereses del público usuario, así como promover el sano desarrollo de estos sectores con el propósito de extender la cobertura de sus servicios a la mayor parte posible de la población.

Crédito. Referirse a la palabra crédito, conlleva el indagar para conocer diversas definiciones para comprender claramente el tema. A continuación, se presentan las más significativas:

"Es la parte activa de la relación jurídico-obligatoria. Esta relación, es la relación "crédito-deuda". El crédito en este sentido es la acreencia, el derecho que tiene el acreedor de exigir el pago de la deuda a su deudor; es pues, la deuda aún no cobrada" (Torres, 2010)

El crédito es un préstamo de dinero que el Banco otorga a su cliente, con el compromiso de que, en el futuro, el cliente devolverá dicho préstamo en forma gradual (mediante el pago de cuotas) o en un solo pago y con un interés adicional que compensa al Banco por todo el tiempo que no tuvo ese dinero (mediante el prepago). (BANCAFACIL, 2015)

Los créditos son dados por los distintos bancos, efectuando el propósito de entregarlos a sus clientes, sobre todo personas y empresas.

De acuerdo con el Banco Mundial (BM), México está por debajo de la media de América Latina en el acceso al crédito. El nivel de penetración crediticia, es decir, el crédito a personas y empresas, que tiene México de 32\% respecto al PIB lo ubica en el lugar 116 de 169 países, por debajo de naciones como Botsuana, Surinam o Senegal, de acuerdo con un estudio realizado por el Banco Mundial.

Los recientes datos del Banco Mundial, con cifras al cierre de 2015 revelan que en los últimos 10 años esta proporción en México se ha duplicado al pasar de $16.2 \%$ en 2005 a $32.7 \%$ en 2015 . La última vez que México alcanzó niveles superiores a los $30 \%$ fue en 1994, previo a la crisis y de ahí tocó un mínimo de $13 \%$ en el 2001.

El Fondo Monetario Internacional (FMI) destacó en su reciente análisis sobre "Profundización financiera en México" que el nivel de penetración crediticia ha sido "excepcionalmente bajo" durante décadas, pero también ha demostrado que puede recuperarse. (Catro, 2017)

Lo anterior permite abordar el término referido a la Educación Villavicencio Financiera, por el cual se justifica la investigación hacia la comunidad universitaria de Villavicencio Colombia, ya que cumplen con la característica de ser un consumidor financiero, relacionado al contexto que le rodea y que el mismo, le lleva a solicitar, algunos de los servicios que ofrece la banca múltiple, para satisfacer sus necesidades de corto y mediano plazo. 
Los servicios financieros mencionados anteriormente, en ocasiones no son conocidos e infortunadamente, es escaso el conocimiento del consumidor financiero respecto a lo que implica obtener un crédito a través de algunos de los intermediarios expuestos con anterioridad. El ensayo 'The Anatomy of Ignorance: Diagnoses from Literature' de los expertos de la Universidad de Harvard, Devjani Roy y Richard Zeckhauser, ha analizado cómo la ignorancia se ha convertido en un factor influyente en la vida social, las cuestiones políticas y, cómo no, en las decisiones económicas.

A la hora de tomar una decisión financiera, uno de los peligros de la falta de conocimiento es que la realidad no tenga nada que ver con las posibilidades que la persona ha contemplado. Por ejemplo, en el caso de un pequeño inversor, esta tendencia es realmente delicada ya que una mala decisión, en la que no se han tenido en cuenta todos los factores que entran en juego, puede hacer que los ahorros se esfumen. En este panorama, la educación financiera es vital para no caer en errores que puedan afectar al futuro de las personas y de sus familias. (Financiera, 2017).

\section{Educación Financiera}

Para la OCDE (La Organización para la Cooperación y el Desarrollo Económico), la educación financiera es definida como "el proceso por el cual los consumidores/inversores financieros mejoran su entendimiento de los productos, conceptos y riesgos financieros y, mediante información, instrucción y/o asesoramiento objetivos, desarrollan las habilidades y la confianza para llegar a ser más conscientes" (García, Grifoni, \& López, 2013), lo que significa que, los consumidores deben atender a los riesgos que implica adquirir una deuda, y para ello deben informarse adecuadamente para así tomar decisiones que les permitan mejorar su bienestar económico.

En otras palabras, la educación financiera es el proceso que consiste en el desarrollo de habilidades y capacidades para administrar el dinero con base al conocimiento de productos financieros, la correcta planeación financiera y la práctica del ahorro, con ello, permita tomar decisiones acertadas sobre las finanzas personales $\mathrm{o}$ profesionales en el cumplimiento de los objetivos a corto, mediano y largo plazo.

Cuando se carece de una educación financiera, se refleja en una mala planeación financiera de los ingresos y gastos, ya que la población al desconocer los servicios financieros que existen busca alternativas informales de ahorro o crédito como las tandas, solicitar dinero con un tercero que cobra por arriba intereses fuera de los autorizados por la banca, y guardar dinero en casa por un tiempo, sin darse cuenta de que el dinero pierde valor al no invertirlo en algún instrumento de inversión.

El bienestar económico que desea la población, no se llega a cubrir del todo a razón de su escaso conocimiento sobre los servicios financieros, el entorno económico en el que se desenvuelven con una cultura del "endeudamiento", y el carente sentido de responsabilidad al 
contraer una deuda, que si bien, solventa su situación apremiante, pero, adquieren otra que no podrán cubrir con el paso del tiempo.

Con esto, podemos percatarnos de que las finanzas personales, es un tema que la población desconoce, y es uno de los más importantes, puesto que, si se analizara de manera más profunda la forma en la que ahorra, invierte y gasta el dinero, el impacto en el bolsillo de los ciudadanos sería totalmente distinto. Es de suma importancia implementar programas que fomenten una cultura financiera perspicaz, donde tanto gobierno y población sean capaces de dominar temas como este.

\section{Materiales y Métodos}

En la presente investigación se tomó a la población juvenil universitaria de Villavicencio Colombia. Para calcular el tamaño de la muestra se consideró un porcentaje de error del $10 \%$ un nivel de confianza del $95 \%$ que genera el tamaño de la muestra siendo de 59 estudiantes.

El instrumento que se aplicó cuenta con 18 ítems que permiten identificar el nivel de conocimiento sobre educación financiera por parte de los jóvenes de Villavicencio Colombia, misma que se aplicó y se validó a través del coeficiente de Alpha de Cronbach que fue de .857 por lo que se considera que los resultados son altamente confiables.

\section{Resultados y Discusión}

Conforme al objetivo establecido al inicio de este trabajo de investigación, medir el nivel de conocimiento sobre educación financiera entre los estudiantes de nivel licenciatura Villavicencio Colombia, se alcanzaron los resultados subsiguientes:

\section{Género}

Para este caso en particular se encuentra que el $41 \%$ de los estudiantes son hombres y el 59\% son mujeres, un fenómeno que es muy recurrente en las licenciaturas de las ciencias económicoadministrativas, en donde el sexo femenino tiene mayor participación.

\section{Actividad}

En esta parte se identifica que el $62 \%$ de los encuestados solamente se dedican a estudiar, Sin duda el tener el respaldo económico familiar, facilita el que los estudiantes puedan formarse, sin embargo, se observa que existe un $38 \%$ de los estudiantes que trabaja y esto también representa una situación en donde los jóvenes empiezan a tener un acercamiento con los temas financieros.

\section{Estado Civil}

Para el caso del estado civil se encontró que el $90 \%$ de los estudiantes se encuentran solteros lo que representa una gran ventaja para continuar con su formación e iniciar en el ámbito laboral; por otra parte, se encuentra que solo el $3 \%$ se encuentra casado y el $7 \%$ restante vive en unión libre reflejando que, a pesar de ya tener responsabilidades de tipo económico, han decidió continuar sus estudios.

\section{Hijos}


En esta parte se encontró que el $88 \%$ de los estudiantes no tiene hijos aun lo que representa otra ventaja para continuar con los estudios y poder iniciar la vida laboral, sin embargo, también se encontró que el $12 \%$ ya tiene hijos, lo que puede representar un gran reto, debido a las responsabilidades económicas que conlleva tener hijos y que tienden a ser exponenciales, pero a pesar de ello se mantienen realizando los estudios universitarios.

\section{Vivienda}

En esta investigación se encontró que el $64 \%$ de los estudiantes vive con sus papás, lo cual asegura de cierta manera estabilidad económica para continuar con su educación, el $22 \%$ de los encuestados muestra que han tenido que desplazarse de su hogar, ya sea porque la Institución Educativa se encuentra en un lugar diferente al de su residencia o busca mejores oportunidades, lo cual deja ver que se vuelven personas más independientes que saben administrar sus recursos y con ello son más responsables, el $14 \%$ vive con amigos o pareja, al compartir vivienda con otra persona fuera de casa no sólo nos refleja independencia sino que también son capaces de solventar los gastos de manera equitativa.

\section{Decisiones financieras}

Podemos ver que el $76 \%$ de las decisiones financieras son tomadas por los rangos más altos dentro del modelo tradicional familiar, son decisiones cuidadosamente analizadas por integrantes experimentados en el ámbito, el 19\% de los encuestados toman sus propias decisiones, aunque es un porcentaje menor es una cifra significativa pues nos refleja la presencia de educación financiera, apenas el $4 \%$ de las decisiones son tomadas por la pareja y en un mínimo $2 \%$ ésta cuestión financiera pasa a manos de otro familiar.

\section{Fuentes de ingresos}

El $72 \%$ de los encuestados dependen económicamente de sus padres, sólo el $13 \%$ tienen ingresos propios, hay quienes reciben apoyo económico de algún familiar siendo ellos el $4 \%$, pero sólo un $6 \%$ tienen ingresos mediante un negocio propio, es decir, la cantidad de emprendedores es muy baja, la cifra es muy preocupante ya que no todos se atreven a iniciar un negocio propio, aquí es donde se hace énfasis a la importancia de la educación empresarial, y el 6\% restante obtiene ingresos por medios variados.

\section{Registro de actividades financieras}

Sin duda, llevar un registro de las actividades financieras nos asegura mejor control de nuestras finanzas, las cifran nos arrojan un $28 \%$ de encuestados que lo hacen todo el tiempo por lo que debería asegurarse que administran bien sus ingresos y egresos, el $33 \%$ lo hace frecuentemente, el $13 \%$ lo hace de manera regular, es decir, no son constantes, el 19\% lo hace con poca frecuencia y el $7 \%$ en definitiva no tiene entre sus planes el hacer registro de sus actividades financieras.

\section{Rubros de registro de actividad financiera \\ Veamos ahora las principales razones por las que nuestros encuestados hacen un registro de sus actividades financieras, la}


razón más popular es porque quieren saber en qué han gastado representando ellos el $59 \%$, ahora bien, tenemos un empate de $24 \%$ entre quienes quieren conocer sus ingresos y las deudas que deben cubrir, apenas el $20 \%$ se preocupa por crear un fondo de ahorro, nuestro $22 \%$ restante hace un presupuesto siendo ésta la principal razón por la que deberían hacer un registro de las actividades económicas, un buen presupuesto nos ayuda a saber cuánto dinero se recibe en el hogar, saber cuánto y en qué se gasta el dinero, nos permite ajustar los gastos de acuerdo con los ingresos, fijar metas de ahorro para lograr los sueños y prepararse mejor para las emergencias, es decir, un presupuesto es la forma más completa y efectiva de administrar el capital.

\section{Meta económica}

Si bien es cierto que cada individuo tiene metas diferentes, aquí comprobamos que mientras que el $51 \%$ se preocupa por invertir para después obtener frutos, es decir, instalar un negocio, el $32 \%$ ha respondido que su principal meta económica es la adquisición de una casa, el $6 \%$ tiene como meta concluir sus estudios, mientras que el $2 \%$ prefiere un auto, el 9\% restante tiene metas diferentes, las cuales podrían ser unas vacaciones o ampliar su historia académica con alguna especialización.

\section{Medios de pago}

El pago en efectivo es el predominante en este caso con un popular $90 \%$, dejando muy por debajo el uso de las tarjetas de crédito al $6 \%$, y un menor $4 \%$ cuando de tarjetas de débito hablamos, algunas otras formas de pago han sido nulas en nuestros resultados, tales como pago con cheque, trasferencias electrónicas y otros medios.

\section{Compras fuera del presupuesto}

Como ya habíamos mencionado, un presupuesto nos ayuda a tener un mejor control de los ingresos y egresos, pero $¿$ en serio nos ajustamos al presupuesto que hacemos? Sólo un 4\% de nuestras encuestas reflejan el seguimiento adecuado de un presupuesto, todas las demás frecuencias demuestran que no sabemos ajustarnos.

\section{Gastos no planeados}

Todos los días pueden presentarse imprevistos, oportunidades para adquirir algún bien o servicio o simples necesidades por cubrir, pero ¿de dónde obtenemos el dinero para pagar por ello? De acuerdo a los resultados de esta encuesta el 59\% cubre esos gastos con sus ahorros, lo cual muestra una buena cultura financiera, el 29\% logra hacerlo con ingresos regulares, el $6 \%$ usa tarjetas de crédito, aunque debemos tener cuidado con las famosas "letras chiquitas", el 4\% recibe apoyo económico de familiares y solamente el $2 \%$ pide préstamo a amigos, sea cual sea el modo de cubrir dichos gastos debemos tener cuidado para no excedernos.

\section{Dinero a final de mes}

Para éstos resultados podemos crear 3 grupos de personas: el primero; a quienes a final de mes le sobra dinero $(33 \%)$; el segundo, personas que no les sobra e incluso les falta dinero $(61 \%)$ y quienes se exceden en los gastos a tal grado que necesitan pedir préstamos y por 
consecuencia adquieren una deuda mayor, si no somos cuidadosos con las finanzas, las deudas pueden acumularse $y$ acumularse a un punto donde sea casi imposible salir.

\section{Importancia de ahorrar}

Sería preocupante no pensar en ahorrar, pero en este caso la respuesta es muy positiva, ya que es un término considerado muy importante.

\section{Ahorro}

La pregunta es muy fácil de responder, incluso se puede hacer casi de inmediato, nuevamente la respuesta más popular es en sentido positivo, ya que un $82 \%$ ha respondido que, si destina parte de sus ingresos a un ahorro, el $16 \%$ que no ahorra esperemos que no lo hagan porque son inversionistas y ese $2 \%$ que no respondió ojalá comience a hacerlo.

\section{Medios de ahorro}

Cuando se trata de ahorrar, existen diversas maneras para hacerlo, algunas pueden ser formales como es en; bancos (18\%), cajas de ahorro (8\%) u otras como inversiones $(4 \%)$, pero también están las informales, esos métodos curiosos pero funcionales en su mayoría de casos como pueden ser: Alcancías $(57 \%$ y más popular), guardar el dinero en cajas o en medio de un libro (12\%) o las famosas tandas $(2 \%)$. Siempre hay que tomar en cuenta que la mejor manera de hacer crecer el dinero es que esté en constante movimiento, es decir, generando intereses.

\section{Porcentaje destinado al ahorro}

Muchos economistas mencionan que el mejor porcentaje de ahorro es a partir del $10 \%$ pero estamos conscientes de que en ocasiones puede no ser tan fácil, la cultura del ahorro es fundamental pero también debemos ser realistas, a veces la situación no lo permite por más que lo intentemos, jamás debe perderse de vista que el monto o porcentaje ideal de ahorro depende de tus metas, necesidades y situación de vida.

\section{Pedir préstamos}

Todos en la vida hemos pasado por momentos de inestabilidad económica (o pasaremos) pero ¿A quién recurrimos cuando no alcanzamos a cubrir nuestros gastos? El $62 \%$ indicó que este apoyo lo recibe por parte de sus padres, lo cual es una ventaja enorme porque normalmente no es necesario pagar intereses y no hay sanciones en caso de exceder el plazo de pago, lo mismo sucede con quienes piden préstamos a amigos representando el 56\%, el $54 \%$ lo pide a familiares, un $12 \%$ se acerca a instituciones bancarias para dichas transacciones, el $8 \%$ prefiere usar una tarjeta de crédito, el $4 \%$ indicó que obtiene dinero por otros medios $y$ únicamente el $2 \%$ de los encuestados no han pasado por ésta situación, lo cual comprueba que han sabido crear presupuestos y han tenido cuidado de no excederse con las cuentas.

\section{Tipos de créditos}

Es esta pregunta predominan las personas que no cuentan con crédito alguno, arrojándonos un saldo del $72 \%$, tenemos doble empate, el primero; del $12 \%$ entre quienes cuentan con préstamo personal y tarjeta de crédito, y el segundo; del $4 \%$ quienes indican tener crédito automotriz y otros tipos de crédito, 
únicamente el $2 \%$ nos muestra que cuentan con crédito hipotecario.

\section{Puntualidad en pagos}

Contar con tarjeta de crédito puede ser de gran ayuda, pero también puede perjudicarnos si no la usamos de manera correcta, ¿qué dicen nuestra encuesta al respecto? ¿Qué tan puntuales somos a la hora de pagar? El $46 \%$ paga toda la deuda, el $25 \%$ no puede pagar la deuda, el $11 \%$ paga el mínimo, pero paga a tiempo, otro $11 \%$ paga más del mínimo y el $7 \%$ paga cuando puede, recordemos que es un apoyo, pero ser impuntuales en los pagos trae consecuencias.

\section{Razones de solicitud de crédito}

Existen diversas razones para solicitar un crédito, va desde querer invertir en la educación propia (31\%), algo tan inevitable como una emergencia familiar o médica $(17 \%)$, tener el deseo de emprender o mantener un negocio (14\%), querer pagar cuentas pendientes (14\%), o ¿por qué no? comprarse un automóvil para desplazarse más cómodamente $(3 \%)$, todos tenemos necesidades pero es cierto que cada quien tiene prioridades diferentes, depende de cada uno destinar el crédito obtenido. Mientras se emplee para generar algo positivo, debe aprovecharse este apoyo.

\section{Inversiones conocidas}

Los economistas afirman que tener el dinero en constante movimiento nos dará como resultado ganancias, veamos ahora en qué medios lo ponemos a trabajar: sin duda la educación es la mejor inversión que puede hacerse y así lo dejó ver el 34\% de los encuestados, nuestra clase emprendedora representa el 26\% invirtiendo en negocios, otros más se han acercado a Instituciones Bancarias para inversiones a plazo (18\%), algunos han decidido involucrarse en la bolsa de valores $(8 \%)$, un $10 \%$ comenzó a invertir en bienes raíces, área que por cierto es de las más rentables y nuestro final $4 \%$ que aún no se ha dado la tarea de investigar qué tipos de inversiones existen y cuáles les convienen.

\section{Conclusiones}

Con base a los resultados obtenidos de esta investigación dirigida a la comunidad universitaria de Villavicencio Colombia, se percibe que, a pesar de reconocer la importancia de temas financieros para su vida personal, los alumnos carecen del conocimiento pertinente respecto al ahorro y crédito formal, en la mayor parte por la influencia de los padres.

Acorde con los servicios financieros existentes, el ahorro, no obstante que se realiza por gran parte de los universitarios, se lleva a cabo de manera informal, al igual que los créditos, pues en casi todos los casos se recurren a fuentes poco formales como la familia y amigos.

El tema del ahorro para el retiro no es relevante en la población joven no, como se vio en los pues se ve reflejado en los datos obtenidos a través de esta investigación que más de la tercera parte encuestada no cuenta con este ahorro

Por otro lado, se visualiza que gran parte de nuestra muestra no tiene una inversión proyectando que las principales 
razones son por falta de recursos y por falta interés. Asombrosamente la respuesta con menos impacto ha sido la de que no se invierte por falta de confianza en las instituciones bancarias

Según lo ya expuesto y sabiendo que los jóvenes se encuentran en disposición de aprender a manejar sus recursos, el que se fortalezca su educación financiera muestra un avance trascendental, pues a pesar de entender la importancia de esta, carecen de conocimiento o no tienen a su alcance la información de servicios financieros que podrían perfeccionar sus recursos financieros.

\section{Referencias Bibliográficas}

Amezcua García, E. L., Arroyo Grant, M. G., \& Espinosa Mejía, F. (23 de Mayo de 2014). Universidad Veracruzana. Obtenido de https://www.uv.mx/iiesca/files/2014/09/03CA20 1401.pdf

Arboleda, U. S. (2010). Maneja tus Finanzas. Obtenido

de https://www.manejatusfinanzas.com/Loqueneces itassaber/Finanzas/Sistemafinanciero/tabid/77/la nguage/es-CO/Default.aspx

BANCAFACIL. (30 de Noviembre de 2015). BANCAFACIL. Obtenido de http://www.bancafacil.cl/bancafacil/servlet/Cont enido ?indice $=1.2 \&$ idPublicacion $=30000000000$ 00027\&idCategoria $=5$

Banco de México. (2017). Banco de México. Obtenido de http://www.banxico.org.mx/divulgacion/glosario /glosario.html

Banco de México. (2017). Obtenido de http://www.banxico.org.mx/divulgacion/sistemafinanciero/sistema-

financiero.html\#ElSistemadeAhorroparaelRetiro ylasafores
BANXICO. (30 de Agosto de 2013). BANXICO.

Obtenido de http://www.anterior.banxico.org.mx/divulgacion /sistema-financiero/sistema-

financiero.html\#Serviciosfinancieros

Burdillo Vázquez, R. (20 de marzo de 2016). Economipedia. Obtenido de http://economipedia.com/definiciones/ahorro.ht $\mathrm{ml}$

Catro, C. (06 de Febrero de 2017). Dinero en Imagen. Obtenido de https://www.dineroenimagen.com/2017-0206/83260

CONASAR. (26 de septiembre de 2018). Gob.mx. Obtenido de Gob.mx: https://www.gob.mx/consar/que-hacemos

CONDUSEF. (05 de Octubre de 2010). ¿Cuál es tu nivel de cultura financiera? Obtenido de http://www.cnnexpansion.com/mi-

dinero/2010/10/05/conduseftras-la-educacionfinanciera.

Credits, G. (30 de Agosto de 2013). Pro Ahorro. Ahorra dinero, reduce tus deudas, aumenta tu riqueza. Obtenido de http://www.proahorro.com/que-son-las-finanzaspersonales/

Domínguez, F. (17 de Julio de 2017). Forbes, México. Obtenido de https://www.forbes.com.mx/mexico-mejora-eneducacion-financiera-pero-no-lo-suficiente/

ENIF. (Agosto de 2017). Comisión Nacional Bancaria y de Valores. Obtenido de https://www.cnbv.gob.mx/Inclusi\%C3\%B3n/Do cuments/Encuesta $\% 20 \mathrm{Naciona} \% 20 \mathrm{de} \% 20 \mathrm{IF} / \mathrm{Cu}$ adr\%C3\%ADptico\%202016\%20(impresi\%C3\% B3n\%20carta).pdf

ENIF. (2017). Comisión Nacional Bancaria y de Valores MX. Obtenido de Comisión Nacional Bancaria y de Valores MX: https://www.cnbv.gob.mx/Inclusi\%C3\%B3n/Do cuments/Reportes\%20de\%20IF/Reporte\%20de $\% 20$ Inclusion\%20Financiera\%208.pdf

Financiera, B. (28 de Septiembre de 2017). BBVA. Obtenido de BBVA: 
https://www.bbva.com/es/afecta-falta-educacionfinanciera-economia/

Financiera, E. (17 de Mayo de 2017). BBVA. Obtenido de BBVA: https://www.bbva.com/es/que-es-la-inversion/

Financieras, S. I. (2017). Banca fácil. Obtenido de http://www.bancafacil.cl/bancafacil/servlet/Cont enido? indice $=1.2 \&$ idPublicacion $=30000000000$ 00027\&idCategoria $=5$

García, N., Grifoni, A., \& López, J. C. (2013). OCDE. Obtenido de http://www.oecd.org/daf/fin/financialeducation/oecd_caf_financial_education_latin_a mericaes.pdf

González, J. D. (Junio de 2017). Euro Mediterranean Network. Obtenido de http://www.eumed.net/librosgratis/actas/2017/desarrollo-empresarial/36educacion-financiera-en-mexico.pdf

Joehnk, G. \&. (2009). Fundamentos de Inversiones. México: Pearson Education.

Management, M. F. (2015). Obtenido de http://www.mfm.com.mx/wpcontent/uploads/2015/10/Curso-deEducaci\%C3\%B3n-Financiera-MFM.pdf

MAPFRE. (22 de junio de 2011). Seguros y pensiones para todos. Obtenido de https://segurosypensionesparatodos.fundacionma pfre.org/syp/es/seguros/definicion-seguroasegurar/

México, G. (2018). gob.mx. Obtenido de https://www.gob.mx/shcp/articulos/conoce-alas-22-instituciones-que-conforman-el-sectorhacendario? idiom $=\mathrm{es}$

Mnuel, G., \& Seria, E. (2015). Consideraciones sobre la evolución y retos del sistema de ahorro para el retiro. México: Fundación de Estudios Financieros - FUNDEF, A.C.

OCDE. (28 de Septiembre de 2005). GOB. Obtenido de GOB: https://www.gob.mx/cms/uploads/attachment/fil e/83054/Educaci_n_Financiera.pdf

OECD. (28 de Septiembre de 2008). GOB. Obtenido de GOB: https://www.gob.mx/cms/uploads/attachment/fil e/83054/Educaci_n_Financiera.pdf

Peumans, H. (1967). Zona Económica. Obtenido de Zona Económica: https://www.zonaeconomica.com/inversion/defi nicion

Sánchez Alvarado, F. (2017). Repositorio Digital IPN. Obtenido de https://www.repositoriodigital.ipn.mx/bitstream/ 123456789/5395/4/52-4.pdf

Sanciprian, E. (16 de febrero de 2018). IDC on line. Obtenido de IDC on line: https://idconline.mx/fiscal-

contable/2018/02/16/que-es-el-sistema-

financiero-mexicano

Terceño, A., \& Guercio, M. B. (15 de Agosto de 2010). El crecimiento económico y el desarrollo del sistema financiero. Un comparativo. En A. Terceño, \& M. B. Guercio, El crecimiento económico y el desarrollo del sistema financiero. Un comparativo (pág. 34). Tarragona. Obtenido de

http://www.anterior.banxico.org.mx/divulgacion /sistema-financiero/sistema-

financiero.html\#Introduccionalsistemafinanciero

Torres, M. (02 de enero de 2010). Crédito desde el punto de vista jurídico. Obtenido de https://www.gestiopolis.com/el-credito-desde-elpunto-de-vista-juridico/

Unión, C. d. (10 de Enero de 2014). Cámara de Diputados, LXIV Legislatura. Obtenido de http://www.diputados.gob.mx/LeyesBiblio/pdf/5 2.pdf

Uribe, J. D. (2013). El sistema financiero colombiano: estructura y evolución reciente. Revista del Banco de la República, 5. 